\title{
Topical Umbilical Cord Serum for Corneal Epithelial Defects after Diabetic Vitrectomy
}

\author{
Siamak Moradian ${ }^{1,2}$, MD; Marzieh Ebrahimi ${ }^{3}$, PhD; Azade Kanaani ${ }^{1}$, MD; Amir Faramarzi ${ }^{2}$, MD; Sare Safi ${ }^{1}$, PhD \\ ${ }^{1}$ Ophthalmic Epidemiology Research Center, Shahid Beheshti University of Medical Sciences, Tehran, Iran \\ ${ }^{2}$ Ophthalmic Research Center, Shahid Beheshti University of Medical Sciences, Tehran, Iran \\ ${ }^{3}$ Department of Stem Cells, Cell Science Research Center, Royan Institute, Tehran, Iran \\ ORCID: \\ Siamak Moradian: https://orcid.org/0000-0002-5328-7565
}

\section{Abstract}

Purpose: To evaluate the role of topical umbilical cord serum (TUCS) therapy in treating corneal epithelial defects (CEDs) after diabetic vitrectomy.

Methods: In this double-masked, randomized clinical trial, we included 80 eyes of 80 patients who were candidates for vitrectomy due to proliferative diabetic retinopathy complications. In cases of corneal edema obscuring the fundus view during surgery, the corneal epithelium was removed using a 6- $\mathrm{mm}$ trephine and a blade no.15. The day after the surgery, patients were randomly divided into two groups: (1) the TUCS group that received $20 \%$ TUCS six times/day in addition to the conventional treatment of CED and (2) the control group, which was prescribed artificial tears as placebo in addition to the conventional treatment of CED. The rate of healing of CEDs was measured via two maximum linear dimensions perpendicular to each other at the start of therapy and on postoperative days $1-5,7$, and 12 .

Results: Of 80 eyes, 40 were assigned to each treatment group. The mean times to complete CED healing were $2.4 \pm 0.7$ and $3.8 \pm 2.1$ days in the TUCS and control groups, respectively $(P<0.001)$. Persistent CED occurred in two eyes in the control group but in no eyes in the TUCS group.

Conclusion: TUCS therapy may be safe and effective in healing CEDs after vitrectomy in patients with diabetes.

Keywords: Corneal Epithelial Defect; Diabetic Vitrectomy; Topical Umbilical Cord Serum

J Ophthalmic Vis Res 2020; 15 (2): 160-165

\section{Correspondence to:}

Siamak Moradian, MD. Department of Ophthalmology, Labbafinejad Medical Center, Shahid Beheshti University of Medical Sciences, Pasdaran Ave., Boostan 9 St., Tehran 16666, Iran.

E-mail: moradian33195@yahoo.com

Received: 26-10-2018Ａccepted: 28-09-2019

\section{Access this article online}

Website: https://knepublishing.com/index.php/JOVR

DOI: 10.18502/jovr.v15i2.6732

\section{INTRODUCTION}

The removal of corneal epithelium may be necessary to improve a surgeon's visualization of

This is an open access journal, and articles are distributed under the terms of the Creative Commons Attribution-NonCommercial-ShareAlike 4.0 License, which allows others to remix, tweak, and build upon the work non-commercially, as long as appropriate credit is given and the new creations are licensed under the identical terms. 
fundus during vitrectomy. In diabetic patients, abnormal postoperative healing of corneal epithelial defects (CEDs) results in morbidity and prolongs the hospitalization period. Generally, patients with diabetes are at a high risk of developing corneal disorders, a condition known as diabetic keratopathy due to insufficient epithelial adherence to the Bowman's membrane. Significant recurrent corneal erosions can occur after intraocular surgery, particularly vitrectomy, in such patients. ${ }^{[1,2]}$ Treatment options for diabetic keratopathy are limited. Recent studies have reported the effectiveness of topical umbilical cord serum (TUCS) in treating ocular surface disorders, such as neurotrophic keratitis, dry eye syndrome, and persistent CEDs. ${ }^{[3-5]}$ Several growth factors, such as the epidermal growth factor (EGF), acidic and basic fibroblast growth factors (FGFs), platelet-derived growth factor, hepatocyte growth factor, vitamin $A$, transforming growth factor- $\beta$ (TGF- $\beta$ ), substance $P$, insulin-like growth factor-1 (IGF-1), nerve growth factor (NGF), fibronectin, and $\alpha_{2}$-macroglobulin, are present in umbilical cord serum (UCS). Furthermore, concentrations of EGF, TGF-B, and NGF are several times higher in UCS than in peripheral blood. ${ }^{[4,5]}$ We performed this study to investigate whether TUCS may have a beneficial role in healing CEDs after diabetic vitrectomy.

\section{METHODS}

In this double-masked, prospective randomized controlled clinical trial (RCT), we included all patients with type II diabetes who underwent deep vitrectomy because of proliferative diabetic retinopathy (PDR) complications and had corneal epithelial edema during surgery that prompted removal of the corneal epithelium to improve the media clarity. A 6-mm trephine was used to equalize the basic CED size.

The exclusion criteria were a history of herpetic, exposure, or neurotrophic keratitis, previous keratorefractive surgery, significant dry eye, trachomatous keratopathy, immune or nutritional deficiency, a history of autoimmune diseases, lid abnormality, use of glaucoma eye drops or any other eye drop after surgery (except for drops that were used during the study), and pregnancy. Eyes with severe lid edema after surgery were also excluded.

The study protocol was approved by the institutional review board of the Ophthalmic Research
Center of the Shahid Beheshti University of Medical Sciences. This study was conducted according to the Declaration of Helsinki and all participants gave written informed consent before entering the study. This RCT was registered at www.ClinicalTrial.gov (NCT01168375).

Patients in the TUCS group received 20\% TUCS eye drops (diluted with preservativefree artificial tears) six times/day in addition to the conventional treatment (chloramphenicol and betamethasone eye drops four times daily and cycloplegic eye drops three times daily). Patients in the control group received the conventional treatment and a placebo (preservative-free artificial tears), without patching the eye between the drop administrations.

Patients were followed-up on postoperative days $1-5,7$, and 12 . Corneal photography was performed after administration of the fluorescein dye. The longest linear diameter and the longest vertical diameter of the CED area were measured and multiplied to obtain the area of the equivalent rectangle. The rate of healing of CEDs was measured at each subsequent follow-up. The umbilical cord blood was obtained from healthy mothers with uncomplicated cesarean section delivery after screening for parenterally transmitted viral diseases, hepatitis $B$ and $C$, human immunodeficiency virus, cytomegalovirus, and syphilis at the Royan Institute Cord Blood Bank. Briefly, samples that were negative for viruses and microbes were centrifuged at 1,500 rotations/min for $5 \mathrm{~min}$, and the supernatant cells were discarded. No anticoagulants were used in the procedure. Umbilical cord serum was stored at $-20.0^{\circ} \mathrm{C}$ in $5 \mathrm{ml}$ bottles. The maximum storage time was three months. To prepare eye drops, one bottle was opened, and UCS was diluted with preservative-free artificial tears to achieve the $20 \%$ concentration. Samples were then stored at $4.0^{\circ} \mathrm{C}$ for three days. Subsequently, a new bottle was opened, and the old bottle was discarded. Randomization was performed using the random-block permutation method using a computer-generated randomized list. Patients were then assigned to one of the two groups: case or control. A random allocation sequence was performed by a biostatistician. Details of the series were unknown to the study investigators. All study personnel and participants were masked to the treatment throughout the study. TUCS and placebo eye drops were prepared and labeled using codes 
by a non-study ward staff based on the randomization list provided. Only the study biostatistician who had no contact with the study participants was unmasked to the treatment groups.

\section{Sample Size}

In the pilot study, the standard deviations of time to remission in the umbilical cord serum and placebo groups were 0.8 and 2.0, respectively. To achieve an $85 \%$ power in detecting one-day differences between the new intervention and conventional treatment groups with a type-l error of $5 \%$, we needed 40 patients in each group.

\section{Statistical Analysis}

Data are expressed as mean \pm standard deviation, median, range, percent, and 95\% confidence interval $(\mathrm{Cl})$. To evaluate differences between groups, we used the Chi-square test, Fisher's exact test and $t$-test, whenever appropriate. Relationships between age and duration of operation and time to remission were evaluated using Spearman's correlation coefficient. Analyses were performed using SPSS (version 17.0, SPSS Inc., Chicago, IL, USA). P $<0.05$ was considered statistically significant.

\section{RESULTS}

In total, 80 eyes of 80 patients (42 women [52.5\%] and 38 men [47.5\%]), with a mean age of $58.5 \pm 10.3$ (median: 58; range: 34 to 80 ) years, were enrolled in this study. Their mean basic CED size was $15.5 \pm$ 6.5 (median: 14; range: 2 to 33) $\mathrm{mm}^{2}$ the day after the surgery [Table 1].

As demonstrated in Table 2 and Figure 1, the rate of improvement of CEDs was 1.3 days lesser in the case group than in the control group (95\% Cl: 0.62.1, $P<0.001)$.

There was no statistically significant difference between men and women in the rate of improvement $(3.13 \pm 1.74$ in men vs $3.05 \pm 1.73$ in women, $P$ $=0.833)$ in the case and control groups $(P=0.451$ and $P=0.885$, respectively).

Additionally, we found no significant correlation of the age or duration of operation with the overall rate of improvement in either group [Table 3]. Two cases of persistent CED occurred in the control group, but no eyes in the TUCS group showed this complication.

\section{DISCUSSION}

Diabetic vitrectomy typically necessitates pars plana vitrectomy to treat complications of PDR. Corneal epithelial edema and late corneal decompensation can occur during vitreoretinal surgery. ${ }^{[6]}$ Intraoperative corneal epithelial edema causes media haziness; therefore, the surgeon may mechanically scrape the epithelium with a blade to improve the media clarity and visualization of the fundus. Intraoperative removal of the corneal epithelium or accidental surgical trauma to the corneal epithelium results in postoperative CED, and healing of such lesions can be delayed, particularly in patients with diabetes. ${ }^{[2,7]}$ Postoperative CED is a significant cause of morbidity in these patients. Virata et al reported that $45 \%$ of such cases of CEDs persisted for longer than one week and $28 \%$ lasted for four or more weeks. One reported case resulted in a bacterial corneal ulcer. ${ }^{[8]}$ In one study, the overall rate of corneal epithelial debridement during vitrectomy averaged among 55 vitreoretinal surgeons was 17.4\% $\pm 19.0 \%$ and ranged from $0 \%$ to $90 \%,{ }^{[9]}$ although other studies reported this rate to be approximately 14-34\%. ${ }^{[10,11]}$ Both structural (polymegathism and pleomorphism) and functional abnormalities (increased permeability and slower recovery from induced edema) are observed in the diabetic corneal epithelium and endothelium. ${ }^{[12]}$ EGF, ${ }^{[13-16]} F G F$, and interlukin- $6^{[17]}$ stimulate corneal epithelial migration and proliferation. Fibronectin, ${ }^{[18]}$ hyaluronan, ${ }^{[19]}$ laminin, ${ }^{[20]}$ and collagen type IV are the extracellular matrix components that can facilitate epithelial cell migration. Neuronotrophic substances, such as substance P, IGF-I, and NGF promote corneal epithelial migration; topical application promotes corneal wound healing. ${ }^{[21-25]}$ EGF, vitamin $A$, substance $P, \alpha_{2}$-macroglobulin, fibronectin, and acidic and basic FGFs are tear components that play major roles in proliferation, differentiation, and maturation of the corneal epithelial cells. ${ }^{[2,27]}$ Autologous serum contains factors, such as glucose, proteins, calcium ion, vitamin A, EGF, fibronectin, and glycoproteins, that facilitate corneal wound healing. ${ }^{[28-30]}$ Therefore, autologous serum eye drops are used to treat ocular surface disorders. ${ }^{[31]}$ 


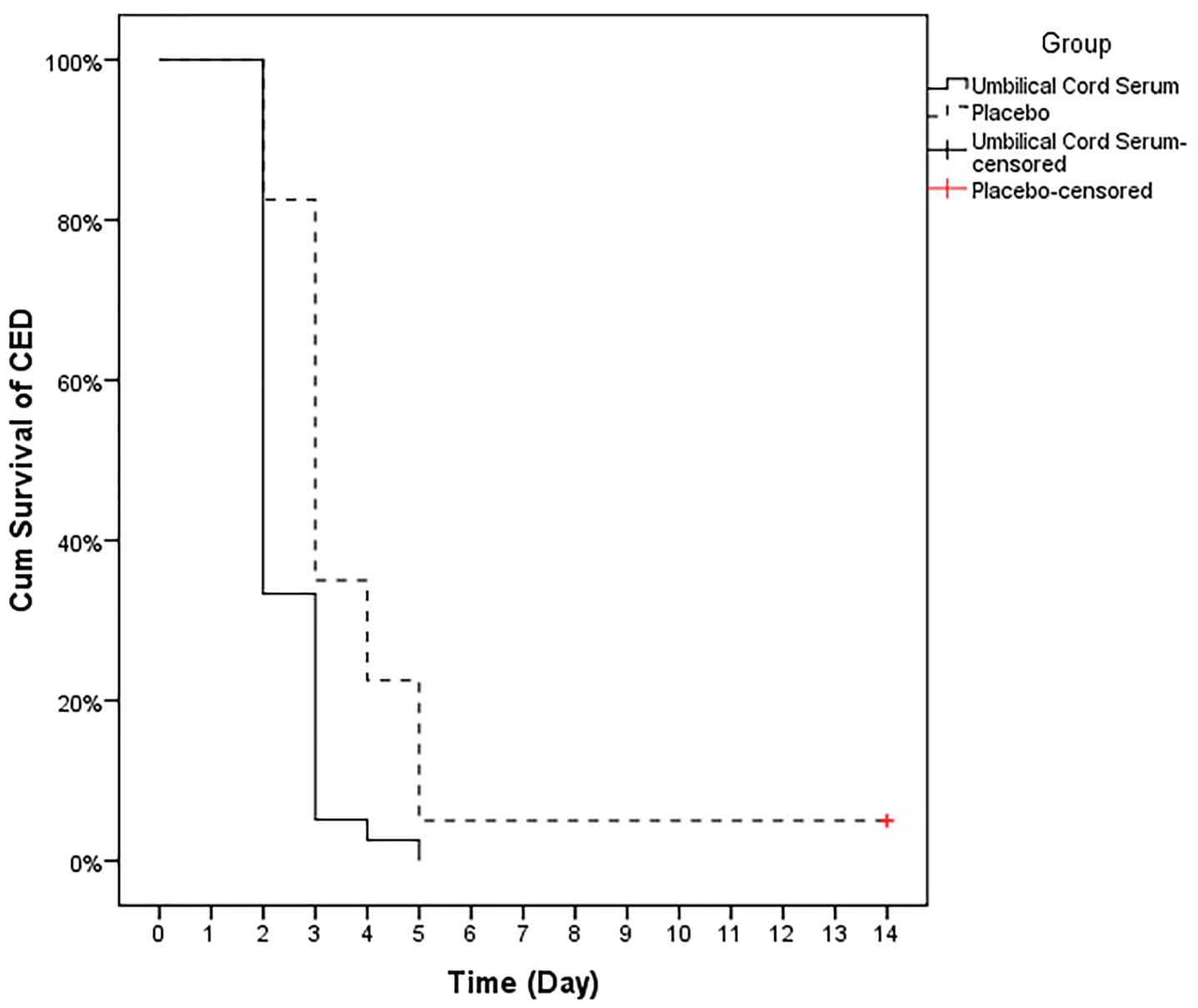

Figure 1. Rate of corneal epithelial defect improvement in the topical umbilical cord serum and control groups. CED, corneal epithelial defect; Cum, cumulative

Table 1. Baseline characteristics of participants

\begin{tabular}{|c|c|c|c|c|}
\hline \multirow[t]{2}{*}{ Variable } & \multirow[t]{2}{*}{ Statistics } & \multicolumn{2}{|c|}{ Groups } & \multirow[t]{2}{*}{ P-value } \\
\hline & & Umbilical cord serum $(n=40)$ & Placebo $(n=40)$ & \\
\hline Age (years) & Mean \pm SD (Range) & $57.6 \pm 9.6$ (34 to 78$)$ & $59.5 \pm 11.0$ (37 to 80$)$ & $0.400^{\dagger}$ \\
\hline Sex & $\mathrm{F} / \mathrm{M}(\mathrm{F} \%)$ & $22 / 55$ (55\%) & $20 / 20(50 \%)$ & $0.654^{*}$ \\
\hline Basic CED Size & Mean \pm SD (Range) & $15.3 \pm 6.2$ (2.3 to 30$)$ & $15.7 \pm 6.8(2$ to 33$)$ & $0.801^{\dagger}$ \\
\hline Operation duration (hours) & Mean \pm SD (Range) & $1.65 \pm 0.62(1$ to 4$)$ & $1.45 \pm 0.50$ ( 1 to 2$)$ & $0.118^{* *}$ \\
\hline
\end{tabular}

†Based on t-test; *Based on Chi-square test; ** based on Fisher's exact test. CED, corneal epithelial defect; F, female; M, male; $\mathrm{SD}$, standard deviation

Table 2. Mean \pm standard deviation (range) of the rate of improvement (days)

\begin{tabular}{|c|c|c|c|c|c|}
\hline Operation & Groups & & & & \\
\hline & Umbilical cord serum $(n=40)$ & Placebo $(n=40)$ & Total & Diff (95\% Cl) & P-value (Between) \\
\hline Vitrectomy & $2.4 \pm 0.7(2$ to 5$)$ & $3.8 \pm 2.1(2$ to 12$)$ & & $-1.3(-2.1$ to -0.6$)$ & $<0.001 \uparrow$ \\
\hline
\end{tabular}

$\dagger$ Based on t-test 
Table 3. Correlation of age and duration of operation in each group with the rate of improvement of corneal epithelial defects

\begin{tabular}{|c|c|c|c|c|c|c|}
\hline \multicolumn{7}{|c|}{ Groups } \\
\hline & \multicolumn{2}{|c|}{ Umbilical cord serum $(n=40)$} & \multicolumn{2}{|c|}{ Controls $(n=40)$} & \multicolumn{2}{|c|}{ Total } \\
\hline & Correlation & P-value & Correlation & P-value & Correlation & P-value \\
\hline Age & 0.205 & 0.211 & -0.004 & 0.979 & 0.073 & 0.522 \\
\hline Operation duration & -0.202 & 0.219 & -0.202 & 0.212 & -0.218 & 0.054 \\
\hline
\end{tabular}

Based on Spearman's correlation coefficient

Autologous serum eye drops are effective in cases of keratoconjunctivitis sicca, ${ }^{[32]}$ severe dry eye, ${ }^{[33,34]}$ superior limbal keratoconjunctivitis, ${ }^{[35]}$ persistent CED, and neurotrophic keratopathies. ${ }^{[36]}$ Higher concentrations of EGF, vitamin $A$, acidic and basic FGFs, fibronectin, NGF, substance $P$, and $\alpha_{2}$-macroglobulin, which are present in UCS, make it more effective than autologous serum in treating ocular surface disorders. ${ }^{[31]}$ Recently, Vajpayee et al reported that TUCS eye drops resulted in faster healing of persistent CED refractory to medical treatments compared to autologous serum drops. ${ }^{[37]}$ Yoon et al found that the application of TUCS effectively treated dry eye and persistent CED due to neurotrophic keratitis. ${ }^{[3-5]}$ Autologous serum preparation requires collection of blood at regular intervals from patients and may induce discomfort. It is difficult to obtain blood samples from patients with a poor general condition or blood dyscrasia, but abundant serum can be drawn from the umbilical vein at once. Many patients can benefit from this sampling approach, which minimizes discomfort. ${ }^{[5]}$ In this study, we examined the effects of TUCS therapy on improvement of CED after diabetic vitrectomy. Our study showed that TUCS hastened the healing of CEDs after vitrectomy relative to the conventional therapy. Persistent CED occurred in two cases in the control group but in no cases in the TUCS group.

The limitations of our study were the absence of dry eye tests before surgery and unavailability of precise measurement of CEDs using Image $\mathrm{J}$ or other digital measurement modalities.

In conclusion, TUCS may significantly accelerate the rate of improvement of CEDs after diabetic vitrectomy and reduce the risk of persistent CED. Further studies with larger sample size are needed.

\section{Financial Support and Sponsorship}

None.

\section{Conflicts of Interest}

There are no conflicts of interest.

\section{REFERENCES}

1. Bright Bill FS, Myers FL, Bresnick G. Post vitrectomy keratopathy. Am J Ophthalmol 1978;85:651-655.

2. Foulds GN, Thoft RA, Perry HD, Tolentino FI. Factors related to corneal complications after closed vitrectomy in diabetics. Arch Ophthalmol 1979;97:1076-1077.

3. Yoon KC, Im SK, Park YG, Jung YD, Yang SY, Choi J. Application of umbilical cord serum eye drops for the treatment of dry eye syndrome. Cornea 2006;25:268272.

4. Yoon KC, Heo H, Im SK, You IC, Kim YH, Park YG . Comparison of autologous serum and umbilical cord serum eye drops for dry eye syndrome. Am J Ophthalmol 2007;144:86-29.

5. Yoon KC, Heo H, Jeong IY, Park YG. Therapeutic effect of umbilical cord serum eye drops for persistent epithelial defect. Korean J Ophthalmol 2005;19:174-178.

6. Bright Bill FS, Myers FL, Bresnick GH. Post vitrectomy keratopathy. Am J Ophthalmol 1978;85:651-655.

7. Fribery TR, Doran DL, Lazenby FL. The effect of vitreous and retinal surgery on corneal endothelial cell density. Ophthalmology 1984;91:1166-1169.

8. Virata SR, Kylstra JA, Singh HT. Corneal epithelial defects following vitrectomy surgery using hand-held, sew-on, and noncontact viewing lenses. Retina 1999;19:287-290.

9. Friberg TR, Ohji M, Scherer JJ, Tano Y. Frequency of epithelial debridement during diabetic vitrectomy. Am J Ophthalmol 2003;135:552-554.

10. Oyakawa RT, Schachat AP, Michels RG, Rice TA. Complications of vitreous surgery for diabetic retinopathy: I. Intraoperative complications. Ophthalmology 1983;90:517-521.

11. Chung H, Tolentino FI, Cagita VN, A losta J Refojo MF. Reevaluation of corneal complications after closed vitrectomy. Arch Ophthalmol 1988;106:916-919.

12. Cisarik-Fredenburg P. Discoveries in research on diabetic keratopathy. Optomery 2001;72:691-704. 
13. Brazzell RK, Stern ME, Aquavella JV, Beuerman RW, Baird L. Human recombinant epidermal growth factor in experimental corneal wound healing. Invest Ophthalmol Vis Sci 1991;32:339-340.

14. Schultz GS, Chegimini N, Grant M, Khaw P, Mackay A. Effect of growth factors on corneal wound healing. Arch Ophthalmol 1992:70:60-66.

15. Maldorado BA, Furcht LT. Epidermal growth factor stimulates integrin-mediated cell migration of cultured human corneal epithelial cells on fibronectin and arginineglycerine-aspartic acid peptide. Invest Ophthalmol Vis Sci 1995;36:2120-2126.

16. David T, Rieck P, Renard G, Hartmann C, Courtois Y, Pouliquen $Y$. Corneal wound healing modulation using basic fibroblast growth factor after excimer laser photorefractive keratectomy. Cornea 1995;14:227-234.

17. Nishida T, Nakamura M, Mishiana H, Otori T, Hikida M. Intreleukin 6 facilitates corneal epithelial wound closure in vivo. Arch Ophthalmol 1992;110:1292-1294.

18. Ohji M, Mandarinol, Sundar Raj N, Thoft RA. Corneal epithelial cell attachment with endogenous laminin and fibronectin. Invest Ophthalmol Vis Sci 1993;34:24872492.

19. Nakamura M, Nishida I, Hikida M, Otori T. Combined effects of hyalophane and fibronectin on corneal epithelial wound closure of rabbit in vivo. Curr Eye Res 1994;13:385388.

20. Maldonado BA, Furcht LT. Involvement of integrins with adhesion promoting, heparin- binding peptides of type IV collagen in cultured human corneal epithelial cells. Invest Ophthalmol Vis Sci 195;36:364-372.

21. Nishida T, Nakamura M, Ofuji K, Reid TW, Manoes MJ, Murphy GJ. Synergistic effects of substance $p$ with insulinlike growth factor-1 on epithelial migration of the cornea. $J$ Cell Physiol 1990;169:159-166.

22. Nokamura M, Ofujik, Chikama T, Nishida. Combined effects of substance $P$ and insulin- like growth factor-1 on corneal epithelial would closure of rabbit in vivo. Curr Eye Res 1997;16:275-278.

23. Tan $\mathrm{MH}$, Bryars $\mathrm{J}$.Use of nerve growth to treat congenital neurotrophic corneal ulceration .Cornea 2006;25:b352b355.

24. Bonni S, Lambiase A, Rama P, Caprioglio G, Aloe L. Topical treatment with nerve growth factor for neurotrophic keratitis. Ophthalmology 2000;107:1347-1351

25. Lambiase A, Rama P, Bonni S, Caprioglio G, Aloe L. Topical treatment with nerve growth factor for corneal neurotrophic ulcers. N Eng J Med 1998;338:1174-1180.
26. Ohashi Y, Motokura M, Kinoshita Y, Mano T, Watanabe $\mathrm{H}$, Kinoshita S, et al. Presence of epidermal growth factor in human tears. Invest Ophthalmol Vis Sci 1989;30:1879_ 1887.

27. Van Setten G, Teno T, Tervo K, Tarkkanen A. Epidermal growth factor (EGF) in ocular fluid: presence, origin and therapeutic consideration. Acta Ophthalmol 1992;202:54-59.

28. Dua HS, Gomes JA, Singh A. Corneal epithelial wound healing. Br J Ophthalmol 1994:78;401-408.

29. Geerling G, Maclennan S, Hartwing D. Autologous serum eye drops for ocular surface disorders. $\mathrm{Br} \mathrm{J}$ Ophthalmol 2004;88:1467-1474.

30. Tsubota K, Goto E, Shimmura S, Shimazaki J. Treatment of persistent corneal epithelial defect by autologous serum applications. Ophthalmology 1999;106:1984-1987.

31. Nobel BA, Loh RSK, Maclennan S, Pesudovs K, Reynolds $A$, Bridges LR, et al. Comparison of autologous serum eye drops with conventional therapy in a randomized controlled crossover trial for ocular surface disease. $\mathrm{Br} \mathrm{J}$ Ophthalmol 2004:88:647-652.

32. Fox RI, Chan R, Michelson J, Belmont JB, Michelson PE. Beneficial effect of artificial tears made with autologous serum in patients with keratoconjunctivitis sica. Arthritis Rheum 1984;27:456-461.

33. Poon AC, Geerling G, Dart JK, Fraenkel GE, Daniels JT. Autologus serum eye drops for dry eyes and epithelial defects: clinical and in vitro toxi city studies. $\mathrm{Br} \mathrm{J}$ Ophthalmol 2007;85:1188-1197.

34. Tananuvat N, Daniell M, Sullivan LJ, Yi Q, McKelvie P, McCarty DJ. Controlled study of the use of use of autologous serum in dry eye patients. Cornea 2001;20:802806.

35. Goto E, Shimmura S, Shimazski J, Tsubota K. Treatment of superior Limbic kerato conjunctivitis by application of autologous serum. Cornea 2001;20:807-810.

36. Matsumoto Y, Dogru M, Goto E, Ohashi Y, Kojima T, Ishida $\mathrm{R}$, et al. Autologous serum application in the treatment of neurotrophic keratopathy. Ophthalmology 2004;111:11151120.

37. Vajpayee RB, Mukerji N, Tandon R, Sharma N, Pandey $\mathrm{RM}$, Biswas NR, et al. Evaluation of umbilical cord serum therapy for persistent corneal epithelial defects. $\mathrm{Br} \mathrm{J}$ Ophthalmol 2003;87:1312-1316. 